\title{
Stage II Uterine Corpus Cancer AJCC v8
}

National Cancer Institute

\section{Source}

National Cancer Institute. Stage II Uterine Corpus Cancer A/CC v8. NCI Thesaurus. Code C139805.

Stage II includes: T2, N0, M0. T2: Uterine corpus carcinoma or carcinosarcoma with tumor invading the stromal connective tissue of the cervix but not extending beyond the uterus. Does not include endocervical glandular involvement. NO: No regional lymph node metastasis. M0: No distant metastasis. (AJCC 8th Ed.) 